\title{
Cuatro preguntas que interrogan el enunciado de Literaturas Regionales: Conversatorio con Ariel Castillo y Mario Jursich
}

Four questions that interrogates the statement of Regional Literatures: A talk with Ariel Castillo and Mario Jursich

\author{
Mario Andrés Páez Ruiz \\ Psicólogo \\ Universidad Autónoma de Bucaramanga \\ Grupo de investigación Violencia, Lenguaje y Estudios Culturales \\ Semillero de investigación Sujeto y Psicoanálisis \\ Docente de la Facultad de Ciencias de la Salud \\ Universidad Autónoma de Bucaramanga. \\ mpaez2@unab.edu.co
}

Artículo recibido el 16 de octubre del 2015 Aprobado el 26 de octubre del 2015

\section{Resumen}

La escritura y publicación de este artículo de revisión de tema obedece a dos propósitos concretos; el primero, realizar un texto que recoja parte de las memorias relativas a los temas discutidos durante el desarrollo de la celebración anual de U Libro 2015, lo cual para el caso de este escrito corresponde a la temática de Literaturas Regionales. En segundo lugar, plantear una discusión elaborada a partir de los aportes de Ariel Castillo y Mario Jursich, expuestos mediante su participación en un conversatorio que tuvo lugar en dicho evento. Para tales efectos, el cuerpo de este artículo se encuentra ordenado en el abordaje de cuatro preguntas emergentes de la conversación con Castillo y Jursich en U Libro 2015. 
Palabra Clave: Literaturas Regionales.

\begin{abstract}
Writing and publishing of this article due to two specific purposes; first, to make a text containing some of the reports concerning the issues discussed during the development of the annual celebration of U Libro 2015, which in the case of this paper corresponds to the theme of "Regional Literature". Second, raise an argument made from the contributions of Ariel Castillo and Mario Jursich, exposed by participating in a conversation that took place in this event. To this purposes, the body of this article is ordered in addressing four emerging questions from the conversation with Castillo and Jursich in U Libro 2015.
\end{abstract}

Key word: Regional Literature

“No existe literatura tomada como un conjunto de obras de valor asegurado e inalterable, caracterizado por ciertas propiedades, intrínsecas y compartidas. Cuando en el resto del libro se usen las palabras "literario" y

"literatura" llevaran una especie de invisible tachadura para indicar que realmente no son las apropiadas pero que de momento no cuento con nada mejor. (...) No hay ni obras ni tradiciones literarias valederas, por sí mismas, independientemente de lo que sobre ellas se haya dicho o se vaya a decir”. Terry Eagleton

“Una Introducción a la Teoría Literaria” (Segunda edición en español de 1998: 22 - 23).

\title{
Introducción:
}

La obra literaria, en sus diferentes estructuras narrativas (novela, cuento, ensayo, poesía, fabula, leyenda o mito), ofrece no solo la oportunidad de contemplar la estética de su contenido, sino también recursos que aplican en la reflexiones sobre cuestiones de orden pedagógico, al igual que sobre la identidad y la memoria de los pueblos, los acontecimientos sociopolíticos coyunturales en la actualidad, y la historia de las tendencias occidentales en filosofía y psicología entre otros asuntos. La celebración anual de U Libro, que toma por espacio la Universidad Autónoma de Bucaramanga, representa una oportunidad para recordar esta premisa; la literatura en este escenario no se restringe a ser expuesta como un tema de contemplación sobre el arte de escribir bellamente, sino que es tomada como plataforma de diálogos que confrontan y articulan diferentes perspectivas, intereses, preguntas y formas de entender el arte. En este contexto se piensa por ejemplo, sobre la función de la literatura en la universidad, su importancia para la memoria histórica de un país en guerra, y el vínculo de su relación con las artes plásticas. 
Es entonces en el compartir de estos diálogos en que aparece el interés por ocuparse de interrogar el concepto de literaturas regionales; esta oportunidad fue facilitada en el conversatorio que tuvo lugar entre Ariel Castillo y Mario Jursich, encuentro desarrollado como parte de las actividades agendadas para esta décima tercera versión de la feria del libro en Bucaramanga, la cual tuvo por núcleo temático el enunciado de Espíritu del Arte.

El tema de las literaturas regionales cobra interés al ser pensado en consideración de las siguientes preguntas: En primer lugar, ¿respetando los límites del rigor conceptual en términos literarios, sin remitirse a una referencia de procedencia geográfica, es posible sostener una categoría tal como Literaturas Regionales? En segundo lugar, ¿enunciar la categoría de Literaturas Regionales, no es acaso hacer mención de un relato de la historia de la literatura de los pueblos más que estrictamente a un concepto literario? En tercer lugar, ¿qué pone de manifiesto una reflexión sobre las Literaturas Regionales al respecto de los actuales criterios de publicación que usa la industria editorial? Y en último lugar, ¿existe una influencia regional sobre los núcleos temáticos a partir de los cuales se escriben textos literarios?

La exposición desarrollada en la presente revisión de tema, corresponde a un esfuerzo por abordar las cuestiones mencionadas, tomando como punto de partida los aportes que en virtud de su experiencia han brindado generosamente Ariel Castillo y Mario Jursich durante el conversatorio en el que han participado, pero también proponiendo un avance al combinar tales aportes con una mirada crítica y otras alusiones pertinentes de traer a colación en el tratamiento de este tema.

\section{Al respecto de estas cuatro preguntas:}

Entrar a precisar definiciones y categorías en el terreno de lo literario ha representado siempre una labor controvertida, la naturaleza estética de la literatura hace considerablemente difícil delimitar unos parámetros decisivos que determinen un límite entre lo literario y lo que cae por fuera de la literatura. Es indudable que a este respecto han habido esfuerzos filosóficos y teorizaciones muy notables que permiten hoy comprender las distinciones entre la novela, el teatro, en ensayo y la poética, aportes de la semiótica cuyos análisis arrojan valiosas luces para comprender las estructuras en que se componen la tragedia, la comedia y la epopeya que respectivamente han emergido bajo la influencia de diferentes épocas (Eagleton, 1996). Sin embargo, a pesar de la antigüedad que pueden tener estas reflexiones, la duda y la incertidumbre se han impuesto de manera constante en el ejercicio de juzgar un texto con pretensiones literarias; para efectos de ilustrar con un ejemplo, llama la atención que en la literatura francesa del siglo XVIII Víctor Hugo y Alexandre Dumas (Padre) no 
fueron siempre vistos con aceptación, al igual que ocurrió con la poesía de Whitman en las letras de la lengua inglesa reconocidas en el mismo siglo ya citado, tuvo que pasar no poco tiempo y algunas transformaciones en los cánones literarios vigentes en Europa y América durante la modernidad, para que la obra de estos escritores fuera leída con el valor suficiente para apreciar en ella sus virtudes literarias.

En este orden de ideas, puede advertirse entonces lo movedizo y por ende lo difícil que resulta proponer categorías literarias; cuesta mucho perfilar una categoría, establecer sobre todo los criterios de exclusión de la misma, las características esenciales que la discriminan de otras categorías. Esto por supuesto puede tornarse más complejo cuando se es consciente de que esta es una labor de índole meramente académica e incluso histórica, pues en realidad pareciera ser que quienes dedican sus esfuerzos a la escritura de textos literarios poco o nada se preocupan porque su legado respete los lineamientos de las categorías vigentes.

Tan solo empezar con el ejercicio de detallar estas ineludibles exigencias, pone ya de manifiesto los retos con que es necesario lidiar antes de ordenar la clasificación de un acervo literario, podría caerse en un acto de ligereza si llegase a formalizarse una categoría literaria sin resolver antes dichas exigencias. Por esta razón, ante un enunciado tal como el de literaturas regionales, emergen las sospechas sobre su rigurosidad conceptual, puede tal vez resultar útil y por tanto seductor, el que esta denominación facilite el ordenamiento de la producción literaria de un país al pensar la misma en virtud de su procedencia geográfico - regional, pero ¿justifica esta utilidad la producción de un concepto? Muy probablemente no, más aun cuando se torna difusa e inestable la resolución de las exigencias que para la conformación de unos límites conceptuales han sido señaladas hasta este punto.

Registrar y contar la historia de los pueblos es una labor que indudablemente invita a la producción literaria a involucrase y comprometerse en tal cometido, bien conocida y apreciada es la función que tiene la literatura en la construcción de la memoria histórica. Entonces, llevando así la reflexión en esta dirección, es plausible afirmar que el enunciado de "Literaturas Regionales" bien remite a la narración de cómo aparece, cómo se hace, y qué destinos tiene la literatura de los pueblos, ahora bien, si es posible aceptar esta premisa, entonces el enunciado en cuestión refiere a la historia de la literatura de los pueblos y más específicamente, a la historia de la literatura enmarcada por delimitaciones geográficas regionales mas no a un concepto rigurosamente planteado; en tal caso las literaturas regionales representan la memoria de una historia, pero no tendrían el propósito ni el deber de definir un orden de clasificación respecto de la estructura narrativa de los textos literarios, ni de la estética propia de las cualidades de los mismos.

Es importante no perder de vista que la idea enmarcada en el párrafo anterior no implica una mirada desdeñosa de aquello que se entiende por literaturas regionales, justamente el propósito de estas líneas recoge la pretensión de señalar el valor posible de distinguir en este polémico enunciado, concerniente al cual ya se 
ha hecho mención del tema de memoria histórica. Diseccionada de la literatura universal con el debido cuidado de guardar la rigurosidad conceptual pertinente, una categoría tal como literaturas regionales ofrece una interesante estrategia metodológica para indagar sobre la relación que de manera consciente o inconsciente, tejen los escritores literarios entre sus contextos regionales y los núcleos temáticos que escogen para el arco argumental de sus obras, o asimismo para revisar si los contextos regionales pueden generar mediante los dispositivos culturales en vigencia, algún tipo de influencia sobre la escogencia o formación de las herramientas narrativas de las cuales se sirve un determinado conjunto de escritores. En la reflexión de estos asuntos se comienza a perfilar un relato sobre cómo se ha tratado al interior de los pueblos y entre los mismos, la cantidad de temas posibles que motivan escritura de un legado literario.

Al respecto de lo inmediatamente anterior, Mario Jursich realiza una evocación de la obra de los hermanos Grimm, cuya recopilación de cuentos a la vez que va siendo trasmitida de una generación a otra y de un pueblo a otro, es también transformada en medio del movimiento que supone este proceso de construcción de una tradición y de un acervo cultural, ahora bien, esta transformación corresponde a las formas en que las influencias regionales matizan los cuentos. En este punto es importante detallar qué debe ser comprendido por influencias regionales; no son estas en términos estrictos una entidad territorial que legisla y orienta la literatura, representan más bien los efectos que acontecen cuando un legado literario hace contacto con el acervo mítico, religioso, moral, ético o sociológico de un pueblo. Volviendo a los hermanos Grimm para ejemplificar esta idea de las influencias regionales, señala Jursich cómo el contenido de violencia y sexo que encontraban estos recopiladores en los relatos originales inmanentes a la tradición oral del folklore rural europeo, fue filtrado con el paso del tiempo hasta reducir tales componentes a una presencia meramente simbólica, sublimados para adaptar así dichas historias al propósito de ser dirigidas a niños como oyentes con fines educativos, o con el objeto de instaurar la cultura, formar recursos psicológicos necesarias ante las exigencias de la vida cotidiana en familia y sociedad, o asimismo las virtudes propias de las civilizaciones modernas (Bettelheim, 1975). Hacer el rastreo de estas condiciones de emergencia en la literatura de los pueblos, es precisamente lo que puede denominarse la realización de un ejercicio analítico de la memoria en el cual aplica metodológicamente el enunciado de literaturas regionales.

La literatura de los pueblos ha sido un referente tomado muy en consideración para el estudio de la conformación de los mismos, un ejemplo diáfano útil para ilustrar esta situación, se hace manifiesto en la formalización moderna de los estudios del lenguaje, específicamente en lo tocante a la filología y lo que con ella se ha hecho en relación con las civilizaciones antiguas, y más concretamente con las civilizaciones precolombinas, sin embargo, no es necesario limitarse a la modernidad para encontrar ejemplos de esta índole, también señala Jursich como en los encuentros que tenían lugar entre los pueblos de las civilizaciones 
antiguas, era la literatura un referente de gran interés, útil para generar aproximaciones y compartir entre si la cosmovisión que tenían los pueblos.

Como ha tratado de explicarse en las pasadas líneas, las influencias regionales son instituciones culturales que filtran, matizan y dan forma en los escenarios que se constituyen al interior de las civilizaciones, a ese patrimonio de la humanidad que se denomina literatura, y que a su vez posee formalizadas por la academia tantas categorías, estructuras narrativas y géneros. Con esto claro ya, aparece otro asunto prudente de distinguir de las influencias regionales, es decir, entra a colación el negocio de la literatura representado por la industria editorial. No se trata en este punto de sugerir algún tipo de acusación o de satanizar el ejercicio de contemplar y poner en marcha la posibilidad de proponer un comercio legítimo que opere con productos literarios, esto en esencia y manejado con criterios trasparentes, podría bien motivar y beneficiar económicamente a quienes dedican sus esfuerzos al oficio de escribir literatura. El problema de estas iniciativas comerciales resulta no esencialmente cuando este negocio vulnera la calidad del contenido y de la estética en la producción literaria, pues las personas son libres de adquirir o rechazar un producto según sus intereses para con el mismo, el problema radica entonces, en que esta industria construya un monopolio que convierta al lector en cliente y pretenda hacer de los escritores vasallos sujetos a tal clientelismo.

Ariel Castillo recuerda a este respecto, las décadas recientes en las cuales quienes querían publicar su obra literaria solo podían hacerlo desde las ciudades capitales, existían entonces, cuenta Castillo, un filtro editorial cuya atención estaba puesta solo en quienes escribían amparados por las diferentes formas del prestigio capitalino, esto excluía deliberadamente a los escritores de provincia cuyo recorrido no había previamente abierto camino entre los lectores o mejor, entre los clientes de la industria editorial ubicada en las ciudades capitales. Los efectos de esta segmentación de la atención y asimismo del apoyo y de la apertura editorial, afirma Castillo, corresponde en parte a la exclusión del folklore popular y del costumbrismo rural y regional del espectro literario que se produce y publica al interior y exterior un determinado país, y en parte también a otro asunto del que Ariel Castillo no hizo mención, pero que podría precisarse acá como la proliferación de un elitismo que hace de un aspecto de la promoción de la literatura, a saber, el aspecto económico, el fin primordial de la misma, en esto, ya no hay lectores, hay clientes, y ya no hay escritores, sino comerciantes que dan forma sin criterio literario a un producto que se vende como si fuese literatura, y todo esto promovido por una industria acaparadora que sin el menor criterio literario, como ya ha sido dicho, le exige y le indica qué escribir al escritor, para finalmente usar de este y de su obra como una herramienta mediática que acaba cobrando un porcentaje mínimo del arduo trabajo efectuado, pues los dividendos de esta industria quedan en su mayoría en manos de sus inversionistas.

Lo que explica el párrafo anterior, finalmente no es una novedad histórica en la proliferación de la 
literatura universal, siempre ha habido elites que favorecen la publicación de un segmento de la producción literaria, mientras por razones de diversa índole que pueden ser políticas, éticas o incluso hasta arbitrariamente caprichosas, excluyen otros segmentos que terminan siendo relegados al anonimato. Es este el caso por ejemplo de tantos cantares de juglaría que durante la época medieval, teniendo un carácter lirico que bien podría hacerles merecedores de una trascendencia literaria, eran reducidos a una categoría bufonesca que les rebajaba al espacio del entretenimiento del vulgo, mientras el teatro cortesano de la tragedia y la comedia Isabelina de la autoría de William Shakespeare, tomaba en cambio su lugar en los anales de los refinados, apreciados y financiados movimientos artísticos ingleses, que luego pasaron a la elevada memoria y condecoración de la literatura universal.

La lección que aporta esta discusión relativa a los criterios aplicados en razones de publicación por la actual industria editorial, obedece a que según lo expuesto, resulta justificado permanecer advertidos sobre los riesgos que implica no el hecho de comerciar con la producción literaria, sino de reducir la producción literaria a un mero ejercicio mercantilista. Sin embargo, es relevante notar que lo más nefasto de no permanecer atento a estos riesgos no es propiamente un efecto de tipo económico, lo cual como reconocen muchos escritores, en los días actuales ya es una realidad que marca una importante pauta en la producción literaria, lo más peligroso de dichos efectos consiste en aquello que Ariel Castillo se esforzó en concienciar entre sus interlocutores, es decir, en cercenar del alcance popular un valioso legado literario solo por asuntos relacionados con la insuficiente razón de la identidad provincial de tal legado.

Restaría por tratar un último asunto, pero este tendrá lugar en el espacio dedicado a la exposición de las conclusiones, y corresponde a la última pregunta que introduce este escrito y que a su vez abre el desarrollo del mencionado y siguiente apartado.

\section{Conclusiones}

Una de las preguntas que ha motivado el trabajo reflexivo consignado en este texto, concede mediante su resolución el aporte de una posible conclusión relativa a lo que ha sido discutido en el escenario que dispuesto en la celebración de U Libro 2015, ha dibujado los contornos del tema para esta publicación. Tal pregunta puntúa lo siguiente: ¿Existe una influencia regional sobre los núcleos temáticos a partir de los cuales se escriben textos literarios?

Al evocar una situación vivida en su infancia al lado de su madre, el genial Charles Chaplin escribe lo siguiente en las primeras páginas de su Autobiografía publicada en el año de 1964: 
En aquella oscura habitación del sótano de Oakley Street mi madre encendió en mi la luz más benigna que jamás haya conocido el mundo, la que ha dado a la literatura y al teatro sus temas más grandiosos y ricos: el amor, la compasión y la humanidad.” (Chaplin, 1964: 29).

Sumando las palabras de Chaplin a los aspectos tratados en el apartado central de este documento, se puede vislumbrar que las diferencias que existen entre las producciones literarias de los pueblos son tan solo una cuestión asociada a los arcos argumentales de los relatos y los elementos circunstanciales de los mismos, por lo general, la memoria de todos los pueblos es preservada en estructuras narrativas comunes (mito, leyenda, fabula, cuento, novela), sin embargo, en este punto común sobresale aquello que Chaplin enuncia; es en esencia el drama de lo humano el acervo que ofrece un tema para la literatura, y el tratamiento literario de este tema aquello que a su vez ha inspirado la escritura de tantos legados que no pierden su vigencia con el paso del tiempo ni se ven limitados a la imposición de los linderos geográficos, esto es precisamente lo que ha hecho de la literatura un patrimonio humano de carácter universal.

En consideración de lo anterior, atender a la rigurosidad académica autoriza y hace prudente afirmar que las influencias regionales pueden aportar a la producción literaria formas (que pueden referirse al uso del lenguaje) y circunstancias que sirven en la perfilación de un contenido, pero sin duda le preexiste a esta influencia la inquietud creadora del escritor y la interrogación de este ante su propia humanidad y por la experiencia de la vida misma, ya que la literatura no es solo un ejercicio de memoria, sino que como muchos escritores lo han mencionado en repetidas ocasiones (Bettelheim, 1975; Celis, 2009; Magallanes, 2014), es también un ejercicio que milenariamente ha servido a la especie humana en el arte de narrarse a sí misma a partir de la estética y demás recursos del lenguaje, y asimismo en la disciplina ética de comprender y significar la experiencia de la vida.

\section{Referencias:}

Bettelheim, B. (1975). "Psicoanálisis de los Cuentos de Hadas". De la traducción al castellano de Silvia Furió. Segunda edición de 2001 incluida en la colección Biblioteca de Bolsillo. Barcelona: Editorial CRÍTICA.

Castillo, A; Jursich, M. (2015). "Encuentro de Literaturas Regionales”. Conversatorio celebrado el jueves 27 de Agosto de 2015 en el marco de la décimo tercera Feria del Libro U Libro 2015. Moderadora Carolina Romero Saavedra. Bucaramanga: Universidad Autónoma de Bucaramanga.

Chaplin, C. (1964). Mi Autobiografía. Tomado de la primera edición traducida al castellano por Julio Gómez 
de la Serna, publicada en el año de 2014 por el Grupo Editorial Penguin Random Hause, S.A.U. Barcelona, España. (Pp. 29).

Celis, C. (2009). "La Construcción del Sí Mismo a partir del Discurso Narrativo como un Proceso Semiótico”. Cuadernos del CUYANCO. No. I. Grupo de Investigación Cultura y Narración en Colombia. Trabajo escrito bajo la dirección de PhD Ana Cecilia Ojeda Avellaneda. Bucaramanga: Editorial de la Universidad Industrial de Santander, Maestría en Semiótica, Escuela de Idiomas, Facultad de Ciencias Humanas.

Eagleton, T. (1983). Una Introducción a la Teoría Literaria. Tomado de la segunda edición traducida al castellano por José Esteban Calderón, México: Fondo de Cultura Económica (1998: 11 - 28).

Magallanes, R. (2014). “Grafomanía Ética y Escrituras de Sî”. Lección Inaugural del Programa Virtual de Literatura de la Universidad Autónoma de Bucaramanga. Primer Cuatrimestre del año 2014. Bucaramanga. Consultado en: http://unabvirtual.edu.co/images/archivos/leccion_inaugural_enero2014.pdf

Citar este artículo como: Páez, M. (2015) "Cuatro preguntas que interrogan el enunciado de Literaturas Regionales: Conversatorio con Ariel Castillo y Mario Jursich”. En: Revista La Tercera Orilla (15).

Bucaramanga: Universidad Autónoma de Bucaramanga.

Artículo arbitrado por Yaneth Lizarazo Ortega. Especialista en Metodología de la Enseñanza del Español y la Literatura. Universidad de Pamplona. Directora Académica, Programa de Literatura modalidad Virtual. Directora de La Tercera Orilla. Integrante del Comité U-Libro. 